\title{
Introduction to Sophisticated Instrumentation in Education Through the Use of a Scanning Electron Microscope Simulator
}

\author{
G. Casuccio*, H. Lentz*, S. Kennedy*, C. Staun**, J. Lang**, S. Chumbley***, C. Hargrave***, T. \\ Nolan**** \\ * RJ Lee Group, Inc, 350 Hochberg Road, Monroeville, PA, 15146 \\ ** West Greene School District, RD\#5 Box 36-A, Waynesburg, PA 15370 \\ *** Materials Science and Engineering, and Curriculum \& Instructional Technology, Iowa State \\ University, Ames, IA 50011 \\ **** Oak Ridge National Laboratory, PO Box 2008, Oak Ridge, TN 37831-6064
}

The problem of recruiting and retaining students in the various fields of science continues today. One aspect of the problem can be addressed by providing students with meaningful exposure to relevant modern technology. In addition, technology that is inherently interactive, such as imagebased systems, can be implemented in an exploratory problem solving context.

There are a variety of image/composition-based instruments used in research and industry that could be incorporated into relevant modern science/math/engineering/technology curricula but for expense. Some of these instruments are becoming available over the Internet, but they provide limited access to students and, in general, they are not fully functional. Effective instrument simulation could distribute these resources to all students in classes throughout a school year.

Through a variety of formal and informal outreach efforts over the last decade, and interactions with other researchers through personal contacts and the literature, it has become clear that the scanning electron microscope (SEM) is an excellent instrument to simulate for the purpose of education. It produces sharp (focused) images of objects from low to high magnification, and the images can be viewed using continuous pan and zoom functions. Using such images, the morphology (size, shape, and orientation) of objects and their components can be observed and described. In addition, when equipped with an energy dispersive x-ray spectrometer (EDS) system, elemental chemistry from any selected spot can be acquired. The combination of imaging and microchemistry capabilities of the SEM make it a useful tool in research and industry involving all branches of science.

To assess the potential of an SEM simulator as a part of educational module, the authors were awarded an NSF SBIR grant.[1] The result of this effort is the interactive SEM simulator (called the iSEM) and a prototype educational module. The team members include scientists, curriculum specialists, and high school teachers.

To be successful as an educational tool, the iSEM must simulate the important/critical functions of the SEM and the module needs to be pedagogically sound. The critical functions of the SEM to be simulated are the ability to perform a continuous pan and zoom, to measure the size of objects, to save a file or print a current view. These functions are illustrated in Figure 1. To allow for a significant range of magnification needed for the zoom function requires that the base image be very large, the equivalent of several hundred individual SEM images. The simulator needs to be capable of handling these very large images with little to no lag time. 
The critical elements of the module include an interactive lesson involving problem solving and one or more element from national or state content standards. The module will be self contained and incorporate components such as relevant standards, objectives, instructional delivery, assessment, and related or background information.

The initial module will be developed in a forensics setting. This format makes it relatively simple to construct the module as a problem-solving exercise. The student investigates trace evidence to "solve the crime". The investigation of that trace evidence affords the opportunity to discuss that material in light of it's own science content. For example, solving a crime through the investigation of animal hair provides a segue into possible discussions of: a) the fundamental characteristics of mammals, b) the use of biometrics and statistics, c) variations of the same structure in different species, d) the relationship between form and function of the same structure when the organisms live in different environments, e) the geographic ranges of different mammals, to name a few.

The concept of the iSEM simulator incorporated into an educational module will be illustrated.

\section{References}

[1] This research is being supported by NSF SBIR Contract DMI-0128609.

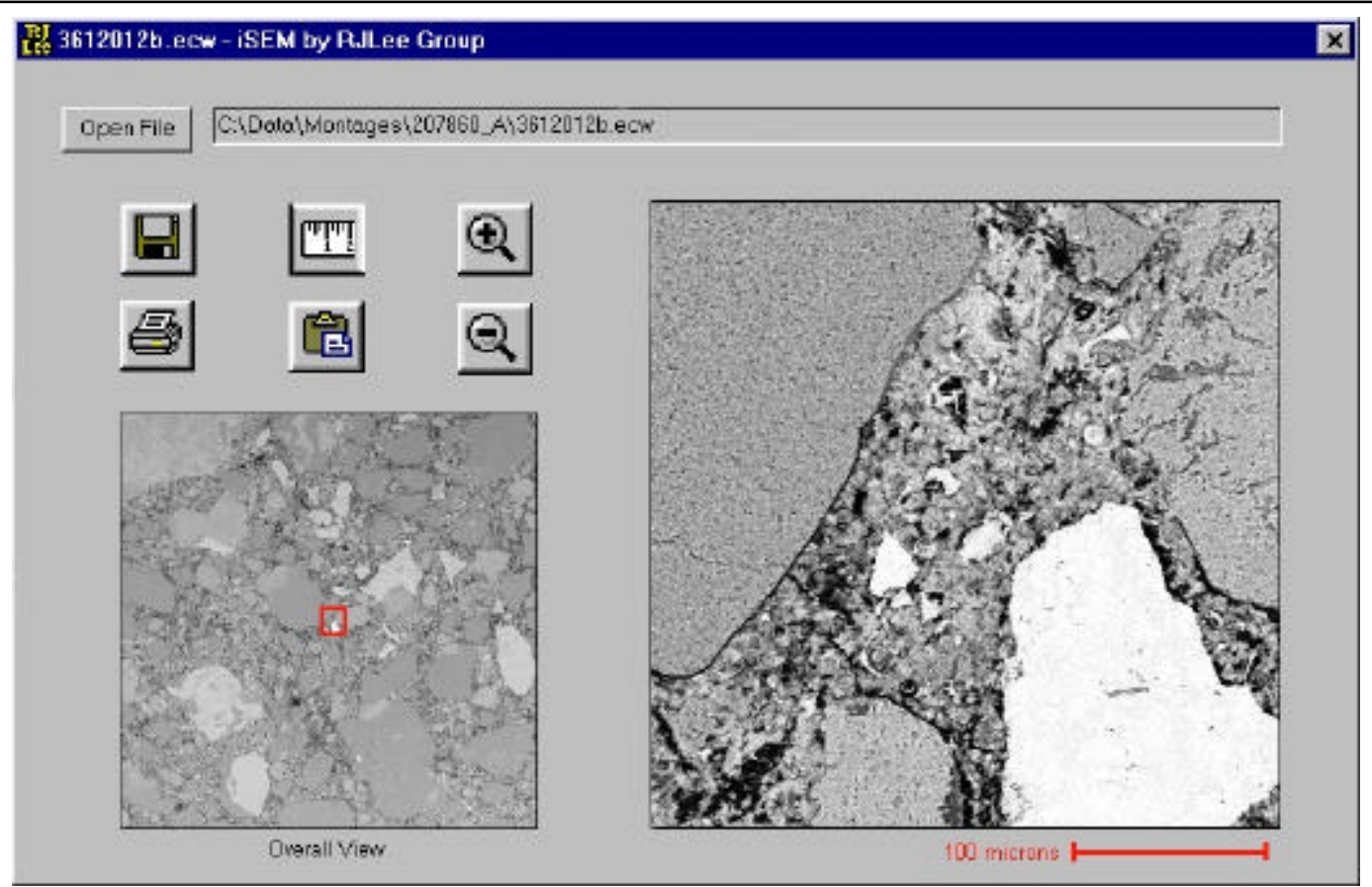

Figure 1 - Prototype interface illustrating the features of the iSEM using a polished section of concrete as the sample. The left image is the "overall view" that displays the entire very large image. The box in that image shows the position and relative magnification of the enlarged image displayed to the right. The position of the box will be controlled using the mouse or a joy stick and the size of the box will be controlled using the + or - magnifying glass. The ruler button will allow the user to measure the length of lines determined using the mouse. The remaining buttons allow the user to save, print, or copy into the clipboard the right image. 